PDFlib PLOP: PDF Linearization, Optimization, Protection

Page inserted by evaluation version www.pdflib.com - sales@pdflib.com 


\title{
IMP Dehydrogenase Inhibitors as Immunomodulators
}

\author{
BEVERLY S. MITCHELL, ${ }^{a, b}$ JENNIFER S. DAYTON, ${ }^{a}$ \\ LAURENCE A. TURKA, ${ }^{\circ}$ AND \\ CRAIG B. THOMPSON $c, d, c$ \\ Departments of Pharmacology $y^{a}$ and Internal Medicine ${ }^{b}$ \\ University of North Carolina \\ Chapel Hill, North Carolina 27599 \\ Departments of Internal Medicine ${ }^{c}$ and Microbiology/Immunology ${ }^{d}$ and \\ Howard Hughes Medical Institute \\ University of Michigan \\ Ann Arbor, Michigan 48109
}

\section{INTRODUCTION}

Inosine monophosphate dehydrogenase (IMPDH; ECl.2.1.14) is a pivotal enzyme in the purine metabolic pathway and regulates the synthesis of guanine ribonucleotides from IMP (FIG. 1). This enzyme has been demonstrated to have higher levels of activity in proliferating cells and has thus served as a target for chemotherapeutic agents. ${ }^{1}$ Mizoribine (bredinin; 4 -carbamoyl-1- $\beta$-D-ribofuranosylimidazolium) is an imidazole nucleoside originally isolated from a soil fungus which has been shown to inhibit IMPDH and to deplete cultured cells of guanine ribonucleotides in vitro. ${ }^{2-4}$ Of interest is the fact that mizoribine has been used clinically in Japan as an immunosuppressive agent $t^{5}$ and has been shown in several experimental systems to have increased effectiveness when used in combination with cyclosporine. ${ }^{6-8}$ In view of the clinical efficacy of this drug, we have investigated the nature of its effects on peripheral blood $T$ lymphocytes in order to answer the following questions: (1) Does mizoribine act by depleting $T$ lymphocytes of guanine ribonucleotides? (2) What are the physiologic effects of guanine ribonucleotide depletion in these cells? and (3) Does 6-mercaptopurine or azathioprine, both inhibitors of purine biosynthesis, inhibit $\mathrm{T}$ cell activation by the same mechanism(s)?

\section{METHODS}

\section{Isolation and Culture of Peripheral Blood T Lymphocytes}

T lymphocytes were isolated from human peripheral blood mononuclear cells using negative selection with a cocktail of monoclonal antibodies as previously described ${ }^{9}$ and were shown to contain greater than $95 \% \mathrm{CD} 2$-positive cells by surface antigen staining before each experiment. Cells were stimulated to proliferate with phorbol myrisitate acetate (PMA) at a final concentration of $3 \mathrm{ng} / \mathrm{ml}$ and ionomycin at 125 $\mathrm{ng} / \mathrm{ml}$ or with anti-CD3 antibody immobilized at a concentration of $1 \mu \mathrm{g} / \mathrm{ml}$ on goat anti-mouse antibody-coated culture dishes. T cells were incubated in RPMI 1640 tissue culture medium containing $10 \%$ fetal calf serum at a concentration of $1 \times 10^{6}$ cells/ 


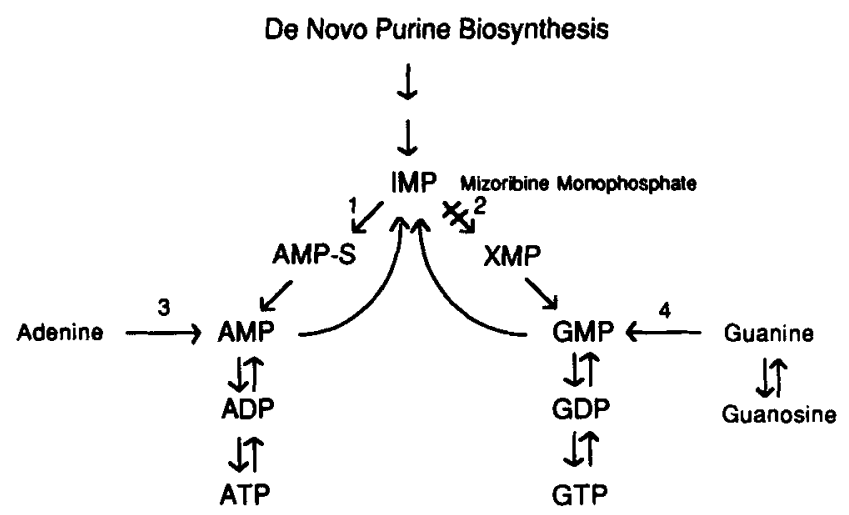

FIGURE 1. De novo and salvage parhways of purine ribonucleotide biosynthesis: 1 , adenylosuccinate synthetase; 2, IMPDH; 3 , adenine phosphoribosyltransferase; 4, hypoxanthine phosphoribosyltransferase.

$\mathrm{ml}$, and proliferation was determined by measuring the incorporation of $1 \mu \mathrm{Ci}$ $\left[{ }^{3} \mathrm{H}\right]$ thymidine into DNA for 6 hours at the end of each culture period. Exogenous guanosine was supplied by adding the combination of guanosine and 8-aminoguanosine. ${ }^{9}$ Guanosine, 8-aminoguanosine, mycophenolic acid, azathioprine, and 6-mercaptopurine were obtained from Sigma Chemical Co. (St. Louis, MO) and added at the final concentrations indicated. Mizoribine was obtained from Dr. K. Fukukawa, Asahi Chemical Co. (Tokyo, Japan).

\section{Cell Cycle Analysis}

$10^{6}$ cells from each experimental condition were fixed in ethanol and incubated with $18 \mu \mathrm{g} / \mathrm{ml}$ propidium iodide and $40 \mu \mathrm{g} / \mathrm{ml} \mathrm{RNase}$ for 16 hours. ${ }^{9}$ DNA content was measured within 24 hours on an Epics C cell sorter (Coulter Electronics, Inc.) and analysis of the cell cycle was performed using the Para 1 software program.

\section{Measurement of ATP and GTP Pools}

Nucleotide pools were measured by high-performance liquid chromatography using a Partisil-10 SAX anion exchange column (Whatman, Clifton, NJ) ${ }^{10}$ Nucleotide levels were quantitated by comparing areas under peaks absorbing at $254 \mathrm{~nm}$ with areas generated by nanomole amounts of pure standards.

\section{RESULTS}

\section{Inhibition of Proliferation and Reduction in GTP Pools in T Lymphocytes Induced by Mizoribine}

Treatment of isolated, purified human peripheral blood $\mathrm{T}$ cells with increasing doses of mizoribine resulted in a progressive decrease in the incorporation of $\left[{ }^{3} \mathrm{H}\right]$ thy- 
TABLE 1. Inhibition of $\mathrm{T}$ Cell Proliferation ${ }^{a}$ Induced by PMA and Ionomycin or CD3 by Mizoribine

\begin{tabular}{|c|c|c|}
\hline $\begin{array}{c}\text { Mizoribine } \\
(\mu \mathrm{g} / \mathrm{ml})\end{array}$ & $\begin{array}{c}\text { PMA } \\
+ \\
\text { Ionomycin } \\
\left(\mathrm{cpm}^{b}\right) \\
\end{array}$ & $\begin{array}{c}\mathrm{CD3} \\
\left(\mathrm{cpm}^{b}\right)\end{array}$ \\
\hline $\begin{array}{c}0 \\
1 \\
5 \\
10 \\
50\end{array}$ & $\begin{aligned} 43,716 & \pm 6,466 \\
32,249 & \pm 1,930 \\
14,918 & \pm 1,096 \\
7,854 & \pm 333 \\
866 & \pm 125\end{aligned}$ & $\begin{aligned} 167,630 & \pm 14,566 \\
32,390 & \pm 2,983 \\
19,192 & \pm 612 \\
7,734 & \pm 656 \\
611 & \pm 73\end{aligned}$ \\
\hline
\end{tabular}

- Proliferation is measured by the incorporation of $1 \mu \mathrm{Ci}$ of $\left[{ }^{3} \mathrm{H}\right]$ thymidine into DNA during the last 18 hours of a 72 -hour culture.

${ }^{b}$ Mean \pm SD of quadruplicate determinations.

midine into DNA, as shown in TABLE 1 . The antiproliferative effect was observed in cells stimulated with monoclonal antibodies to $\mathrm{CD} 3$, as well as in cells stimulated with PMA and ionomycin, indicating that the effect was not dependent on cell membrane signaling events. FIGURE 2 demonstrates that $T$ cell activation by PMA and ionomycin is associated with a progressive rise in GTP levels to a value of 5.8 times that of control levels at 72 hours (panel A). ATP levels increase three-fold over the same interval. Mizoribine at a concentration of $10 \mu \mathrm{g} / \mathrm{ml}$ reduced the total cellular GTP pool by approximately $50 \%$ at each time point of the culture (panel B), but did not reduce
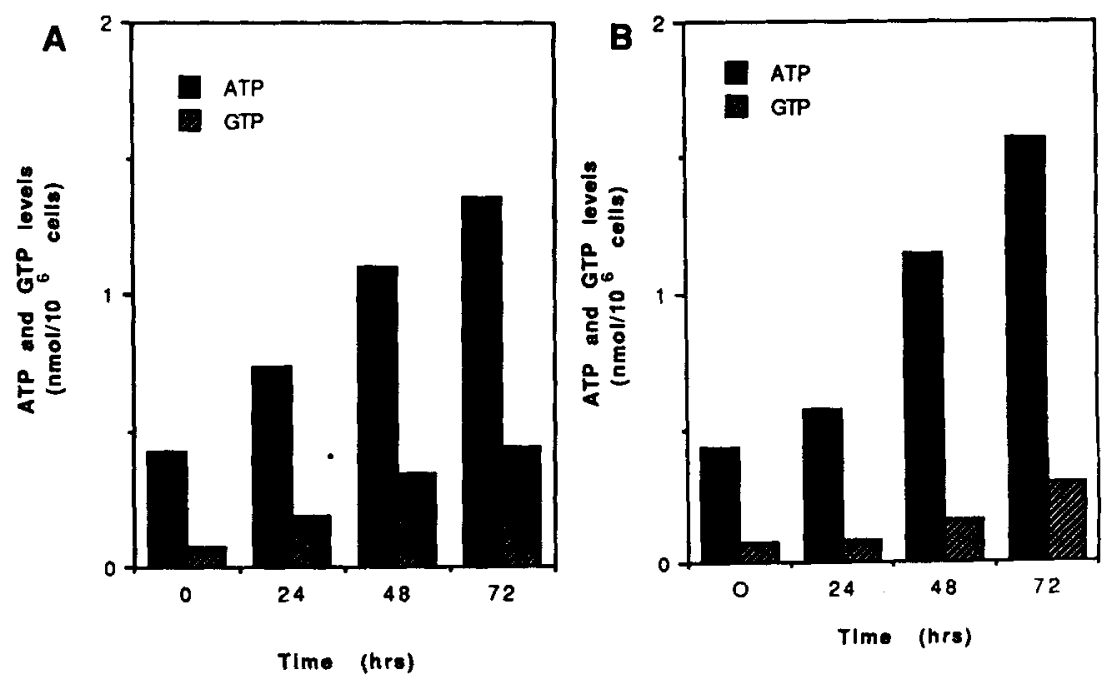

FIGURE 2. Effect of mizoribine on ATP and GTP pools during T cell stimulation with PMA and ionomycin. (A) Stimulation in the absence of mizoribine. (B) Stimulation in the presence of mizoribine. 
TABLE 2. Effect of Mizoribine on Cell Cycle Progression in T Lymphocytes

\begin{tabular}{lcc}
\hline \multicolumn{1}{c}{ Culture Conditions } & $\mathrm{G}_{0}+\mathrm{G}_{1}$ & $\mathrm{~S}+\mathrm{G}_{2}+\mathrm{M}$ \\
\hline Anti-CD3 & 67 & 33 \\
Anti-CD3 + Guo & 55 & 45 \\
Anti-CD3 + Mizoribine & 79 & 21 \\
Anti-CD3 + Mizoribine + Guo & 64 & 36 \\
\hline
\end{tabular}

Note: $T$ lymphocytes were stimulated with anti-CD3 monoclonal antibody alone or in the presence of guanosine $(50 \mu \mathrm{M})$, mizoribine $(2 \mu \mathrm{g} / \mathrm{ml})$, or both compounds.

ATP pools or affect overall cell viability. Restoration of guanine ribonucleotide pools by the addition of $50 \mu \mathrm{M}$ guanosine, a nucleoside that is converted to GMP by the salvage pathway (FIG. 1), increased overall GTP pools to three times the baseline level at 72 hours. ${ }^{9}$ Comparison of the effects of mizoribine with those of azathioprine, 6-mercaptopurine, and mycophenolic acid on $T$ lymphocyte proliferation and purine ribonucleotide pools demonstrated that guanosine completely prevented the inhibition of proliferation induced by mizoribine at concentrations up to and including $10 \mu \mathrm{g} / \mathrm{ml}^{9}$

\section{Effect of Guanine Ribonucleotide Depletion on the Cell Cycle}

In order to determine the effect of guanine ribonucleotide depletion on the cell cycle of $\mathrm{T}$ lymphocytes induced to proliferate from a resting state, we examined the expression of several genes that are sequentially expressed during $T$ cell activation at the mRNA level..$^{9}$ The expression of the c-myc protooncogene, interleukin 2 , and c-myb were unaffected at 6 or 24 hours in the presence of $10 \mu \mathrm{g} / \mathrm{ml} \mathrm{mizoribine.} \mathrm{Cell}$ cycle analysis determined by measuring the DNA content of propoidium iodidestained cells documented a partial block at the $G_{1} / S$ interface of the cell cycle with a mizoribine concentration of $2 \mu \mathrm{g} / \mathrm{ml}$. This block was reversible in the presence of 50 $\mu \mathrm{M}$ guanosine, which repleted GTP pools (TABLE 2). We conclude from these studies that the antiproliferative effects of guanine ribonucleotide depletion are occurring at a point after many of the initial signal transduction events and at or near the point of initiation of DNA synthesis.

\section{Comparison of the Effects of IMP Dehydrogenase Inhibitors with Those of Azathioprine or 6-Mercaptopurine on T Lymphocyte Proliferation and Metabolism}

In order to determine definitively whether the effects of other immunosuppressive agents that interfere with purine biosynthesis also act by depletion of guanine ribonucleotide pools, we compared the effects of mizoribine with those of mycophenolic acid, a known specific inhibitor of IMPDH, 6-mercaptopurine, and azathioprine. All four drugs were found to deplete GTP levels in T lymphocytes stimulated with PMA and ionomycin for 72 hours, and the GTP depletion was reversible in each case with 


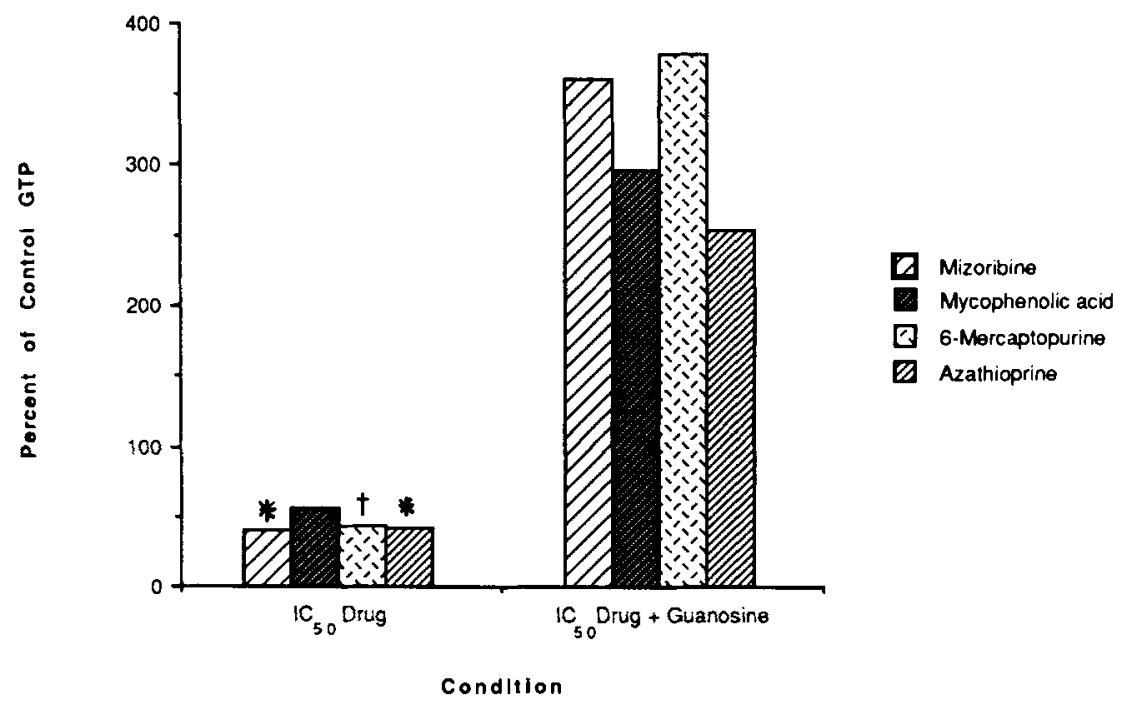

FIGURE 3. Comparison of the effects of $\mathrm{IC}_{50}$ concentrations of mizoribine, mycophenolic acid, azathioprine, and 6-mercaptopurine on GTP pools in purified T lymphocytes after 72 hours of stimulation with PMA and ionomycin. ${ }^{*} p<0.05 ;+p<0.01$. Values are expressed as a percentage of those from untreated controls.

the addition of guanosine (FIg. 3). The inhibition of proliferation seen with both mizoribine and mycophenolic acid was reversed with guanosine; however, the addition of guanosine to 6-mercaptopurine-treated cells only partially reversed the inhibition of proliferation, whereas it significantly increased the inhibition of proliferation induced by azathioprine (FIG. 4). The addition of both adenine and guanosine together was sufficient to restore full proliferation to 6-mercaptopurine-treated cells, but not to azathioprine-treated cells. These data indicate that the inhibitory effects of mizoribine and mycophenolic acid on $\mathrm{T}$ lymphocyte proliferation are mediated primarily by the reduction in guanine ribonucleotide levels, whereas the effects of 6-mercaptopurine are mediated by combined adenine and guanine ribonucleotide depletion and those of azathioprine are unrelated to any effects on the purine ribonucleotide synthetic pathway.

\section{DISCUSSION}

We have determined that mizoribine, a commonly used immunosuppressive drug in Japan, leads to the depletion of guanine ribonucleotide pools in isolated human peripheral blood $\mathrm{T}$ lymphocytes and to concomitant inhibition of proliferation in response to both PMA/ionomycin and anti-CD3 antibody. These findings, in conjunction with the observation that both effects are reversed by the addition of guanosine to the cultures, support the conclusion that inhibition of IMP dehydrogenase by this drug may result in decreased T cell responses to stimularion through the CD3-T cell receptor complex. The precise mechanism leading to the inhibition of proliferation has not yet been established. Our data make it highly unlikely that GTP depletion is 


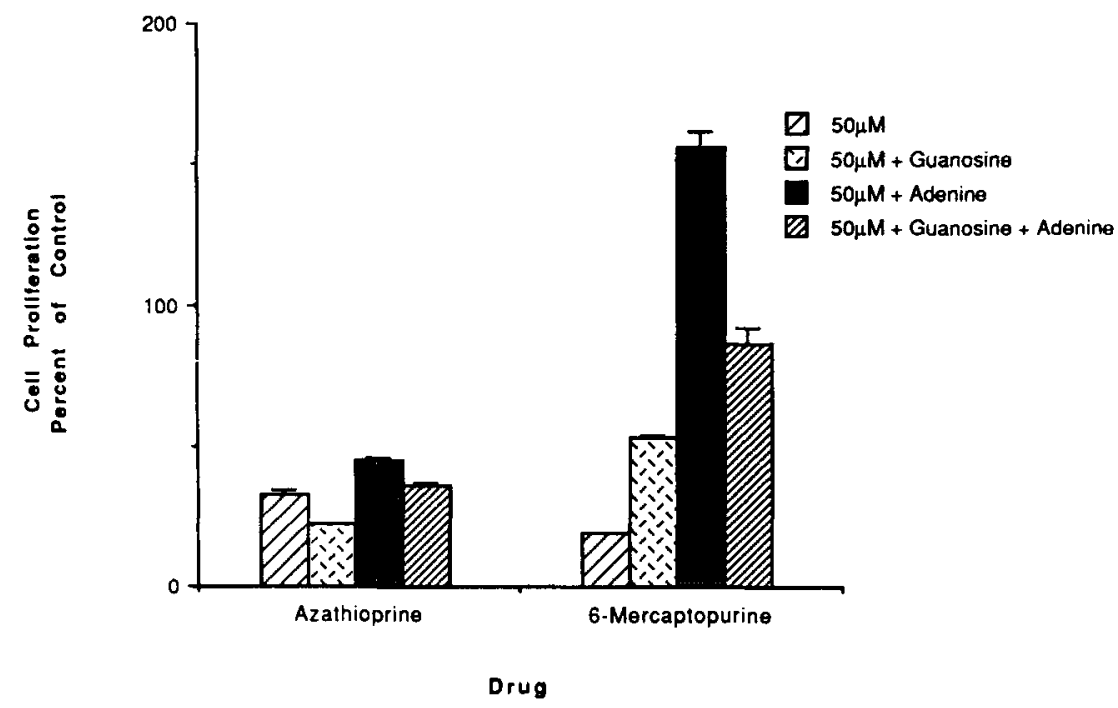

FIGURE 4. Comparison of the effects of ATP and GTP repletion on T lymphocyte proliferation in the presence of $\mathrm{IC}_{50}$ concentrations of azathioprine or 6 -mercaptopurine. Values represent the mean $\pm \mathrm{SD}$ of four determinations.

inhibiting events early in the $\mathrm{T}$ cell activation pathway and support the concept that this metabolic perturbation is affecting one or more events in the DNA synthetic pathway. It has been demonstrated that depletion of GTP is associated with a primary inhibition of DNA synthesis in murine $\$ 49 \mathrm{~T}$ lymphoma cells and that this effect cannot be accounted for by concomitant depletion of the dGTP pool, ${ }^{12}$ but the nature of the GTP requirement remains obscure. Similarly, it is not yet clear why $T$ lymphocytes might be particularly susceptible to the effects of IMPDH inhibition. This selectivity is suggested by the clinical observation that mizoribine at immunosuppressive doses does not have the myelosuppressive effect observed with other inhibitors of DNA synthesis such as methotrexate. Since the activation of peripheral blood T lymphocytes by mitogen or alloantigen involves the transition of a cell from a resting $\left(\mathrm{G}_{0}\right)$ to a proliferating state, it is possible that the requirement for GTP is greater in these cells than in cells already engaged in the cell cycle, although this hypothesis has not yet been tested. Alternatively, the ability of these cells to compensate for GTP depletion induced by inhibitors of de novo purine biosynthesis by using the salvage pathway (FIG. 1) may be less efficient. The use of highly selective inhibitors of IMPDH in vivo in combination with detailed biochemical analysis of the lymphocytes from these individuals should shed further light on these issues.

The observation that IMPDH inhibitors are associared with immunosuppression led us to ask whether either 6-mercaptopurine or azathioprine, which is rapidly metabolized to 6-MP and an imidazole moiety in vivo, ${ }^{13}$ might act by a similar biochemical mechanism. 6-MP is converted to 6-thioIMP, which has been shown to inhibit a number of purine biosynthetic enzymes including IMPDH. ${ }^{14}$ It thus seemed reasonable to ask whether inhibition of proliferation induced by these agents was similar to that induced by mizoribine or by mycophenolic acid. It is clear that neither of these 
agents has any selectivity for IMPDH in intact cells, although both deplete GTP pools. In the case of azathioprine, the mechanism of inhibition of proliferation appears to be completely unrelated to its effect on purine biosynthesis and these data strongly implicate the imidazole moiety as a major factor in inhibiting $T$ cell responsiveness. Of further interest is the observation that azathioprine must be added at or near the start of a 72-hour lymphocyte culture to maximally inhibit proliferation at IC $_{50}$ doses. $^{11}$ This requirement contrasts markedly with the ability of IMPDH inhibitors to act within $\mathbf{2 4}$ hours of addition to already-stimulated $T$ lymphocytes to inhibit proliferation and suggests that there may be an additional role for these compounds once $T$ cell activation has occurred.

Finally, there are substantial data that cyclosporine at low doses may enhance the immunosuppressive effect of mizoribine in experimental animals. ${ }^{6-8,15}$ Our previous experiments have demonstrated an additive effect of cyclosporine on the inhibition of $\mathrm{T}$ cell proliferation by mizoribine and the cumulative data clearly suggest completely different mechanisms of action against $T$ cells. It appears warranted to perform clinical trials using IMPDH inhibitors alone and in combination with reduced doses of cyclosporine in order to determine whether some of the toxic effects of cyclosporine can be attenuated.

\section{SUMMARY}

IMP dehydrogenase is a key enzyme in the de novo pathway of purine biosynthesis and is responsible for catalyzing the first step in the formation of guanine ribonucleotides from inosine monophosphate. Mizoribine, an immunosuppressive agent in widespread clinical use in Japan, has been demonstrated to inhibit this enzyme. We have investigated the effects of mizoribine on human $T$ cell activation. Stimulation of purified human peripheral blood $T$ lymphocytes with phorbol ester and ionomycin leads to a five-fold increase in guanine ribonucleotide levels over 72 hours. The addition of mizoribine to these cultures at concentrations that are achieved in vipo leads to a dose-dependent inhibition of proliferation and concomitant $50 \%$ decrease in guanine ribonucleotide levels, an effect that is reversible with the addition of guanosine, which repletes the GTP pool. Similar effects are seen with direct stimulation via the CD3/T cell receptor complex. Inhibition of proliferation occurs at the $G_{1} / S$ interface of the cell cycle and is additive to that produced by cyclosporine. In order to determine whether inhibition of IMP dehydrogenase is a common mechanism of immunosuppression for drugs such as azathioprine and 6-mercaptopurine that interfere with purine biosynthesis, we compared the effects of these agents on the metabolism of purified $T$ lymphocytes. The results of these studies demonstrate that mizoribine and mycophenolic acid, a highly specific inhibitor of IMP dehydrogenase, inhibit proliferation directly by the depletion of guanine ribonucleotides; 6-mercaptopurine, on the other hand, has a mixed effect on adenine and guanine ribonucleotide pools, whereas azathioprine inhibits proliferation by a mechanism completely independent of its effects on the purine metabolic pathway. We conclude from these studies that inhibitors of IMP dehydrogenase have potential as specific immunosuppressive agents.

\section{REFERENCES}

1. JACKson, R. C. \& G. WEBER. 1975. IMP dehydrogenase, an enzyme linked with proliferation and malignancy. Nature 256: 331-333. 
2. Kоулма, H. \& M. Tsuis. 1983. Genetic and biochemical studies on the activation and cytotoxic mechanism of bredinin, a potent inhibitor of purine biosynthesis in mammalian cells. Biochem. Pharmacol. 32: 3547-3553.

3. Sakaguchi, K., M. Tsuino, M. Hayashi, K. Kawa, J. Mizuno \& K. Hayand. 1976. Mode of action of bredinin with guanylic acid on L5178Y mouse leukemia cells. J. Antibiotics (Tokyo) 29: 1320-1327.

4. Ichikawa, Y., H. Ihara, S. Takahara, K. Takada, G. R. Shrestha, M. Ishibashi, M. Artma, S. SAGAWA \& T. Sonoda. 1984. The immunosuppressive mode of action of mizoribine. Transplantation 38: 262-267.

5. Kusaba, R., O. Otubo, H. Sugimoto, I. Takahashi, Y. Yamada, J. Yamauchi, N. AxTyama \& T. INov. 1981. Immunosuppressive effect of bredinin in the management of patients with renal transplantation. In Proceedings of the European Dialysis Transplantation Associarion 18: 420-425.

6. Amemiya, H., S. Suzuki, H. Watanabe, R. Hayashi \& S. Nirya. 1989. Synergistically enhanced immunosuppressive effect by combined use of cyclosporine and mizoribine. Transplant. Proc. 21: 956-958.

7. Amemiya, H., S. Suzuxi, S. Nirya, H. Watanabe \& T. Kotake. 1988. Synergistic effect of cyclosporine and mizoribine on survival of dog renal allografts. Transplantation 46: 768-771.

8. Aso, K., H. Uchida, K. Sato, K. Yokota, T. Osakabe, Y. Nakayama, M. Ohkubo, K. Kumano, T. Endo, K. Koshiba, K. Watanabe \& N. Kashiwagi. 1987. Immunosuppression with low-dose cyclosporine combined with bredinin and prednisolone. Translant. Proc. 19: 1955-1958.

9. Turka, L. A., J. Dayton, G. Sinclair, C. B. Thompson \& B. S. Mitchell. 1991. Guanine ribonucleotide depletion inhibits $T$ cell activation: mechanism of action of the immunosuppressive drug mizoribine. J. Clin. Invest. 87: 940-948.

10. SIDI, Y. \& B. S. MITCHELI. 1984. 2'-Deoxyguanosine toxicity of B and mature T lymphoid cell lines is mediated by guanine ribonucleotide accumulation. J. Clin. Invest. 74: 16401648.

11. Dayton, J. S., L. A. Turka, C. B. Thompson \& B. S. Mitchell. 1992. Comparison of the effects of mizoribine with those of azathioprine, 6-mercaptopurine, and mycophenolic acid on T lymphocyte proliferation and purine ribonucleotide pools. Mol. Pharmacol. 41: 671-676.

12. Cohen, M. B., J. Maybaum \& W. Sadee. 1981. Guanine nucleotide depletion and toxicity in mouse T lymphoma (S-49) cells. J. Biol. Chem. 256: 8713-8717.

13. Elion, G. B. \& G. H. Hitchings. 1975. Azathioprine. Handb. Exp. Pharmacol 38: 404-425.

14. Wolkerg, G. 1988. Antipurines and purine metabolism. Handb. Exp. Pharmacol. 85: 517-533.

15. Marumo, F., M. Oxubo, K. Yoxota, H. Uchida, K. Kumano, T. Endo, K. Watanabe \& N. KaSHIWAGI. 1988. A clinical study of renal transplant recipients receiving tripledrug therapy-cyclosporine $A$, mizoribine, and prednisolone. Transplant. Proc. 20: 406409. 